\title{
Time-Symmetry Breaking in Hamiltonian Mechanics
}

\author{
Wm.G. Hoover, C.G. Hoover \\ Ruby Valley Research Institute Highway Contract 60, Box 601 \\ Ruby Valley, Nevada 89833 \\ E-mail: hooverwilliam@yahoo.com
}

Received: 11 February 2013; revised: 8 March 2013; accepted: 11 March 2013; published online: 13 March 2013

\begin{abstract}
Hamiltonian trajectories are strictly time-reversible. Any time series of Hamiltonian coordinates $\{q\}$ satisfying Hamilton's motion equations will likewise satisfy them when played "backwards", with the corresponding momenta changing signs: $\{+p\} \longrightarrow\{-p\}$. Here we adopt Levesque and Verlet's precisely bit-reversible motion algorithm to ensure that the trajectory reversibility is exact, with the forward and backward sets of coordinates identical. Nevertheless, the associated instantaneous Lyapunov instability, or "sensitive dependence on initial conditions" of "chaotic" (or "Lyapunov unstable") bit-reversible coordinate trajectories can still exhibit an exponentially growing time-symmetry-breaking irreversibility $\simeq e^{\lambda t}$ Surprisingly, the positive and negative exponents, as well as the forward and backward Lyapunov spectra, $\left\{\lambda^{\text {forward }}(t)\right\}$ and $\left\{\lambda^{\text {backward }}(t)\right\}$, are usually not closely related, and so give four differing topological measures of "local" chaos. We have demonstrated this symmetry breaking for fluid shockwaves, for free expansions, and for chaotic molecular collisions. Here we illustrate and discuss this time-symmetry breaking for three statistical-mechanical systems, [i] a minimal (but still chaotic) one-body "cell model" with a four-dimensional phase space; [ii] relatively small colliding crystallites, for which the whole Lyapunov spectrum is accessible; [iii] a near-continuum inelastic collision of two larger 400-particle balls. In the last two of these pedagogical problems the two colliding bodies coalesce. The particles most prone to Lyapunov instability are dramatically different in the two time directions. Thus this Lyapunov-based symmetry breaking furnishes an interesting Arrow of Time.
\end{abstract}

Key words: reversibility, Lyapunov instability, inelastic collisions, time-symmetry breaking

\section{INTRODUCTION}

The goal we pursue here is improved microscopic understanding of the thermodynamic irreversibility described by the Second Law of Thermodynamics [1]. Unlike the microscopic mechanics which underlies it, the Second Law is strictly irreversible, and applies to macroscopic thermodynamic descriptions of macroscopic processes in which fluctuations are ignored. In Clausius' formulation the Law states that the entropy of an isolated system cannot decrease. The size of the "isolated system" can be anywhere in the range from atomistic to astrophysical so long as the entropy concept makes sense for it. There is no reason to imagine that quantum effects or relativistic effects or gravitational effects are crucial to the Law. Accordingly, we limit ourselves to classical nonrelativistic atomistic models, with short-ranged attractive and repulsive forces, obeying Hamilton's (or, equivalently,
Newton's) time-reversible equations of motion. In particular we emphasize a many-body process for which the apparent irreversibility is especially clearcut. In this example two similar crystalline bodies undergo an inelastic collision in which their kinetic energy is converted to heat. The colliding bodies start out with minimum internal energy and with classical entropy minus infinity. The bodies collide and form a single oscillating liquid drop. Then these oscillations equilibrate. Ultimately the equilibrated drop's internal energy is given by the initial kinetic energy of the two colliding bodies in the frame of the full system's center of mass.

Gibbs' statistical mechanics provides the conceptual basis for thermodynamics, through Liouville's Theorem and Hamiltonian mechanics [2]. In that mechanics, access to all those coordinate-momentum phase-space $\{q, p\}$ states consistent with the initial conditions is typically provided by chaos. Chaos is the sensitive, exponentially-growing time 
dependence of any small perturbation, either forward in time, $\propto e^{\lambda t_{f}}$, or backward in time, $\propto e^{\lambda t_{b}}$. There are two phasespace directions and two Lyapunov exponents for each mechanical degree of freedom. Sets, indicated by braces $\{\ldots\}$, of both "local" [ time-dependent, indicated by $(t)$ ] and "global" [ time-averaged, indicated by $\langle\ldots\rangle$ ] Lyapunov exponents can be used to describe this chaos, with

$$
\left\{\lambda_{\text {global }} \equiv \lambda \equiv\langle\lambda(t)\rangle \equiv\left\langle\lambda_{\text {local }}\right\rangle\right\} .
$$

Details of this exponentially-diverging chaos became available with the advent of fast computers enabling low-cost numerical solutions of the atomistic motion equations. The usual procedure was, and is, to generate a "reference trajectory" and one or more "satellite trajectories", keeping track of the tendency of the satellite trajectories to diverge away from or approach closer to the reference [3-5]. To distinguish this reference trajectory, $\left(q_{0}, q_{d t}, q_{2 d t}, \ldots\right)$ from its reverse, $\left(\ldots, q_{2 d t}, q_{d t}, q_{0}\right)$ we will sometimes term these the "primary" and "reversed" coordinate sets.

The separations of the satellite trajectories from the reference define an orthogonal set of "offset vectors" in the phase space, $\left\{\delta(t) \equiv(q, p)_{\text {sat }}-(q, p)_{\text {ref }}\right\}$. The underlying "molecular dynamics" simulations require five ingredients: forces, initial conditions, boundary conditions, integrators, and diagnostics. Good choices of these five ingredients can give insight into the symmetries and the broken symmetries of Hamiltonian chaos. In what follows we will emphasize "important" particles, those particles making above-average contributions, $\left(\delta q^{2}+\delta p^{2}\right)$ to the offset vector which measures the most rapid divergence of the satellite trajectory from the reference.

Here we select two special Hamiltonian problem types: the dynamics of a single soft disk [6] and the inelastic collision of two many-particle solid bodies [7, 8]. Our interest in the single-particle problem is primarily pedagogical, especially for its apparent ergodicity and for the simplicity of its offset-vector structure. The single-disk "cell-model" problem has only one pair of chaotic offset vectors, a system particularly easy to analyze. Both problem types reveal two interesting aspects of Hamiltonian chaos. First the local Lyapunov exponents have a tendency to pair, corresponding to the forward-backward time reversibility of Hamiltonian motion. The single-disk cell model dynamics apparently illustrates pairing all of the time, once the transient behavior from the initial conditions has decayed. The inelastic collision problems illustrate pairing only most of the time. During the collision process pairing is destroyed.

There is a second consequence of chaos present in both problem types. These Lyapunov exponent pairs illustrate symmetry breaking - for both types, the one-body cell-model problem and the collisional many-body problems. This is because the forward and backward sets of exponent pairs,

$$
\left\{ \pm \lambda^{\text {backward }}(t)\right\} \leftarrow\{q(t)\} \rightarrow\left\{ \pm \lambda^{\text {forward }}(t)\right\},
$$

can be quite different along exactly the same trajectory (both the primary and the reversed orderings) and at exactly the same configuration. This difference reflects the difference between the "past" and the "future". From the qualitative standpoint past and future are about the same for the one-body cell model. Past and Future can and do differ substantially (as described by the Second Law) for the colliding many-body systems treated here.

Demonstrating instantaneous pairing is a numerical challenge. Pairing appears to be present all the time in the simple cell-model problem, with

$$
\begin{aligned}
\lambda_{i}^{\text {forward }}(t) & =-\lambda_{5-i}^{\text {forward }}(t), \\
& \text { and } \\
\lambda_{i}^{\text {backward }}(t) & =-\lambda_{5-i}^{\text {backward }}(t) .
\end{aligned}
$$

On the other hand our numerical work on many-body problems shows that the tendency toward pairing can be defeated by strong localized events. We find that pre-collision pairing is destroyed by energetic collisions of small crystallites, but can apparently recur as the coalesced body equilibrates. We will see clearly that Lyapunov-exponent pairing can be destroyed during the collision process. We also find that a single trajectory's stability can be quite different, forward and backward in time. Forward and backward stabilities, for the same configuration but reversed momenta can and do differ qualitatively. This is a bit surprising. If similar trajectories separate, when propagated forward in time, they correspond to approaching trajectories in the reversed motion. In an idealized perfectly time-reversible situation the first most-positive time-averaged Lyapunov exponent would correspond to the last most-negative exponent if all the geometric data were processed "backward", in the opposite order.

In fact, things are not so simple. Typically $\lambda_{1}^{\text {forward }}(t)$ doesn't correspond to any of the backward exponents. The exponents from a forward processing of coordinate data are not simply related to those from a backward processing. The many-body inelastic-collision problem clearly illustrates this symmetry-breaking exponent pairing. The forward and backward exponent pairs are quite different for exactly the same configuration. In addition there is a qualitative distinction to be seen in the phase-space separation vectors associated with the largest (and smallest) Lyapunov exponents. And the offset-vector differences forward in time don't resemble those with time reversed. These seemingly odd differences invariably emerge when time-reversible Hamiltonian mechanics is applied to highly nonequilibrium situations. We will see that the "important particles" going forward in time can be quite different to those in the reversed motion at the same configuration and with reversed momenta. This symmetrybreaking, with $\left\{\lambda^{\text {forward }}\right\}$ very different to $\left\{\lambda^{\text {backward }}\right\}$ as well as the transient nature of the pairing, $\{+\lambda\}=\{-\lambda\}$, both forward and backward, surprised us and prompted us to write this paper. A second motivation was the relatively-long 
delay in publication of our manuscript [ar $\chi$ iv:1112.5491] "Time's Arrow for Shockwaves; Bit-Reversible Lyapunov and Covariant Vectors ; Symmetry Breaking", submitted to the Journal of Physics A in December of 2011 and finally withdrawn and published in Computational Methods in Science and Technology in early 2013! [9]

This paper is organized as follows. We fix ideas by beginning with the simplest possible one-body Hamiltonian problem. We describe this chaotic problem in Section II, and use it to illustrate Lyapunov instability, the forwardbackward pairing of the local exponents, and symmetry breaking. We follow a simplification suggested by RomeroBastida et alii [10], using Levesque and Verlet's bit-reversible leapfrog algorithm [11] to generate arbitrarily-long perfectlytime-reversible trajectories, both forward and backward in time.

In Section III we consider two larger but still quite manageable problems. In both of them we analyze the inelastic collision of two similar cold crystals. The minimal $N=14$-body simulation of two colliding seven-body hexagons characterizes the stability of the motion in a 56-dimensional $\left\{x, y, p_{x}, p_{y}\right\}$ phase space. Describing any 14-body trajectory in that space involves solving 56 ordinary differential equations. Evaluating the stability of that motion (the 56-dimensional response to perturbations in 56 directions) requires solving $56^{2}$ more differential equations, giving 3192 in all. A more detailed study, following the collisions of two $(4+5+6+7+6+5+4=37)$-particle hexagonal crystallites in their $4 \times 74$-dimensional phase space involves solving $297 \times 296=87,912$ ordinary differential equations. These describe the motion of 296 orthogonal 296-dimensional "offset vectors". The vectors are made orthonormal at the conclusion of every timestep, with a typical collision analysis requiring a few million timesteps.

The interesting topological features connecting an inelastic collision's local Lyapunov spectrum to the phase-space offset vectors can be illustrated by generating a reference trajectory in either of two different ways, [i] bit-reversibly [9-11] or [ii] (slightly) irreversibly, with a highly-accurate fourthorder Runge-Kutta integration. The excellent agreement furnished by these two quite different approaches supports the use of both algorithms. In either case the $4 N$ orthonormalized satellite trajectories are generated with the classic fourthorder Runge-Kutta integrator along with the Gram-Schmidt orthonormalization algorithm.

In Section IV we consider an 800-body problem, where the evolution of the inelastic-collision dynamics takes too long (a few sound-traversal times) for accurate time-reversal using double-precision Runge-Kutta integration. The alternative bit-reversible technique allows us to identify the "important particles" [above-average contributors to $\lambda_{1}(t)$ ] for this highly irreversible process, and provides a clear distinction between the stabilities of the forward and backward (primary and reversed) dynamics. Section V is our Conclusion and
Summary, relating all these time-reversible model results to the irreversibility inherent in the Second Law of Thermodynamics and to microscopic Lyapunov instability.

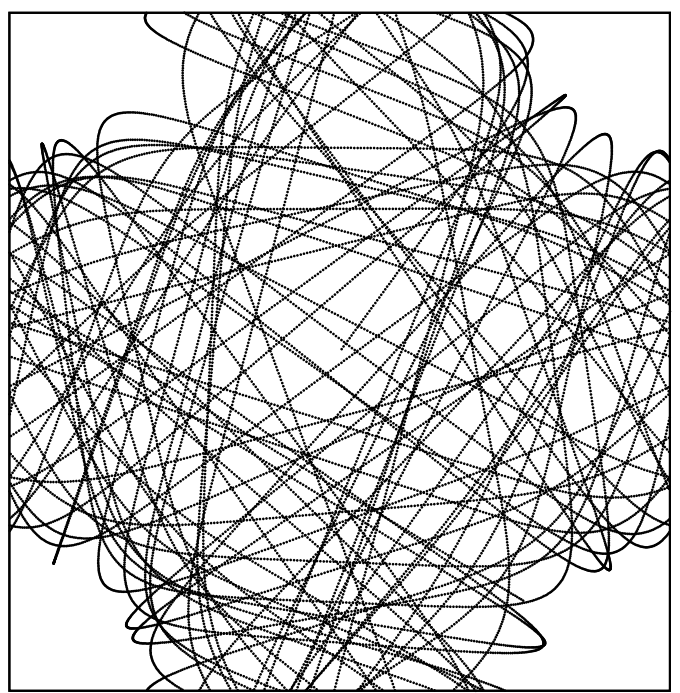

Fig. 1. Sample cell-model trajectory segment for a total time interval $0<t<200$ and $d t=0.001$

\section{ONE-BODY CELL MODEL DYNAMICS}

This simplest chaotic problem is the dynamics of a soft Hamiltonian disk with two degrees of freedom, confined within a periodic square lattice of similar soft-disk scatterers. "Cell models" of this type were studied early in the last century. The corresponding one-particle partition-function models provided semiquantitative "free-volume" estimates for the many-body partition functions characterizing the thensomewhat-mysterious liquid state [12]. The dynamics for this cell-model system occupies a three-dimensional constantenergy volume in the four-dimensional $\left\{x, y, p_{x}, p_{y}\right\}$ phase space. See Figure 1 for a configuration-space view of the dynamics. For this problem, with its periodic boundaries, no attractive forces are necessary. Accordingly, we use a purelyrepulsive potential energy (with numerical integration errors minimized by choosing a pair potential with three continuous derivatives at the cutoff distance of unity):

$$
\phi(r<1)=\left(1-r^{2}\right)^{4} \longrightarrow F(r<1)=8 r\left(1-r^{2}\right)^{3} .
$$

Punctuation of the free-flight regions, by very smooth collisions, enhances the accuracy of the numerical work. For definiteness (so that a diligent reader can reproduce our results in detail) the initial velocity is $(0.6,0.8)$ with the initial coordinates $(0,0)$ in the center of a square-lattice periodic cell. The periodic boundary conditions, $(-1<x, y<+1)$, are imposed by adding or subtracting, if necessary, the cell width 2 at the end of every timestep. Because the spacing between the centers of the fixed nearest-neighbor scatterers 
is 2 , the moving particle interacts with at most one of the fixed particles. For definiteness we choose the initial four offset vectors parallel to the four Cartesian phase-space directions: $\left(x, y, p_{x}, p_{y}\right)$. With fourth-order Runge-Kutta integration, the calculation is insensitive to changes of the timestep, $d t=0.001$, and the length of the offset vectors, $|\delta|=0.00001$. The results described below are obtained by following the dynamics of five separate trajectories, the "reference" trajectory along with four nearby "satellite" trajectories, with the differences defining the four offset vectors $\left\{\delta_{1} \ldots \delta_{4}\right\}$.

To avoid the divergence of the offset vectors that would accompany exponential growth it is usual either [i] to rescale them [3, 4] or [ii] to measure their virtual rates of increase [5], which can be expressed in terms of Lagrange multipliers constraining satellite trajectories to remain at a fixed separation from a reference trajectory. Additional multipliers constrain the directions of the satellite trajectories to remain orthogonal. Numerical work indicates that the positive Lyapunov exponent $\lambda_{1}^{\text {forward }}(+t)$ is accurately paired to its mostly-negative twin $\lambda_{4}^{\text {forward }}(+t)$. Typically this pair of instability exponents, forward in time, is not at all similar to the corresponding pair of "reversed" or "backward" exponents $\left\{\lambda_{1 \text { and } 4}^{\text {backward }}(-t)\right\}$, if the same coordinate trajectory is followed "backward" in time.

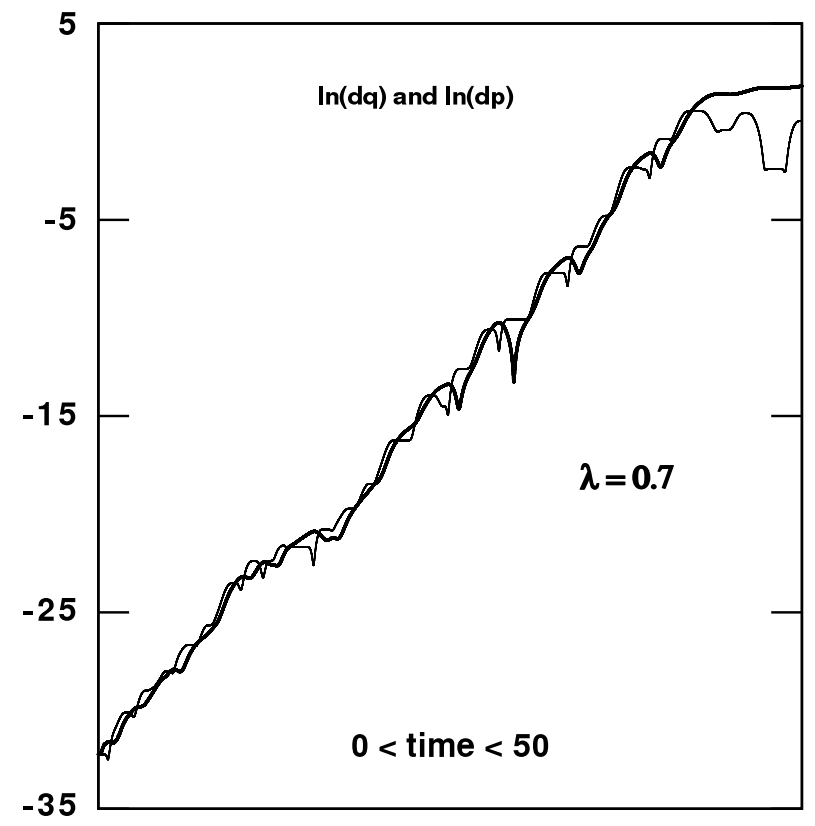

Fig. 2. Growth of small perturbations in the coordinate $q$ and momentum $p$ with time

Despite the pairings, $\lambda_{1}+\lambda_{4} \simeq \lambda_{2}+\lambda_{3} \simeq 0$, the primary exponents, measured in the forward time direction, reflect the past rather than the future. The reversed exponents, measured for the other "backward" time direction, are different. The backward exponents anticipate the "future" rather than reflecting the past. For a typical numerical trajectory segment, which can be followed either forward or backward, see Figure 1. In Figure 2 we see the near-perfect exponential divergence of a small perturbation,

$$
(x, y)_{t=0}=\left(10^{-16}, 0\right) .
$$

The slope gives an estimate of the largest Lyapunov exponent, $\lambda_{1} \simeq 0.7$. By symmetry the smallest exponent is -0.7 so that the time-averaged Lyapunov spectrum is

$$
\{\langle\lambda\rangle\}=\{+0.7,0.0,0.0,-0.7\},
$$

in the four-dimensional phase space of the Hamiltonian motion.

The simplest algorithm characterizing the disk's Lyapunov instability in this space follows the dynamics of a single time-reversible reference trajectory along with four nearby satellite trajectories. The reference-to-satellite vectors $\left\{\delta_{i}\right\}$ are constrained to remain orthogonal at the end of each timestep, maintaining the constant length $\delta \equiv$ 0.00001 . The Gram-Schmidt orthonormalization algorithm first rescales $\delta_{1}$ and then removes the projection of $\delta_{2}$ in the direction of $\delta_{1}$ :

$$
\delta_{2} \longrightarrow \delta_{2}-\delta_{1}\left[\delta_{1} \cdot \delta_{2}\right] / \delta^{2} .
$$

The rescaling operation gives the local value of the Lyapunov exponent $\lambda_{1}$ :

$$
\lambda_{1}(t)=(1 / d t) \ln \left(\delta_{1} / \delta\right) .
$$

Then $\delta_{2}$ is rescaled [giving the second local Lyapunov exponent $\left.\lambda_{2}(t)\right]$ and the projections of $\delta_{3}$ in the directions of $\delta_{1}$ and $\delta_{2}$ are removed:

$$
\delta_{3} \longrightarrow \delta_{3}-\delta_{1}\left[\delta_{1} \cdot \delta_{3}\right] / \delta^{2}-\delta_{2}\left[\delta_{2} \cdot \delta_{3}\right] / \delta^{2} .
$$

Finally $\delta_{3}$ is rescaled, giving $\lambda_{3}(t)$ and $\delta_{4}$ is similarly made orthogonal to $\left\{\delta_{1}, \delta_{2}, \delta_{3}\right\}$ and rescaled to give $\lambda_{4}(t)$. In the end four orthogonal vectors $\left\{\delta_{i}(t)\right\}$ and four local Lyapunov exponents $\left\{\lambda_{i}(t)\right\}$ result.

After a transient time of order $\left(1 / \lambda_{1}\right)$ both the sets of Lyapunov vectors (forward and backward) for the cell model are observed visually to "pair", with about six-figure numerical accuracy:

$$
\lambda_{1}(t)+\lambda_{4}(t) \simeq \lambda_{2}(t)+\lambda_{3}(t) \simeq 0 .
$$

See Figure 3 for a sample sequence obtained with RungeKutta timestep $d t=0.002$ and with the orthogonal vector length $|\delta|=0.00001$. Again, the results are not at all sensitive to either of these choices. Because Hamiltonian mechanics is strictly time-reversible, with all the rates changing sign in a time-reversed simulation, exact pairing, as suggested by our numerical cell-model results, is certainly a plausible property 
of cell-model trajectories. Nevertheless, we will be considering three other chaotic Hamiltonian systems which clearly violate this pairing property, at least some of the time, in the next two Sections.

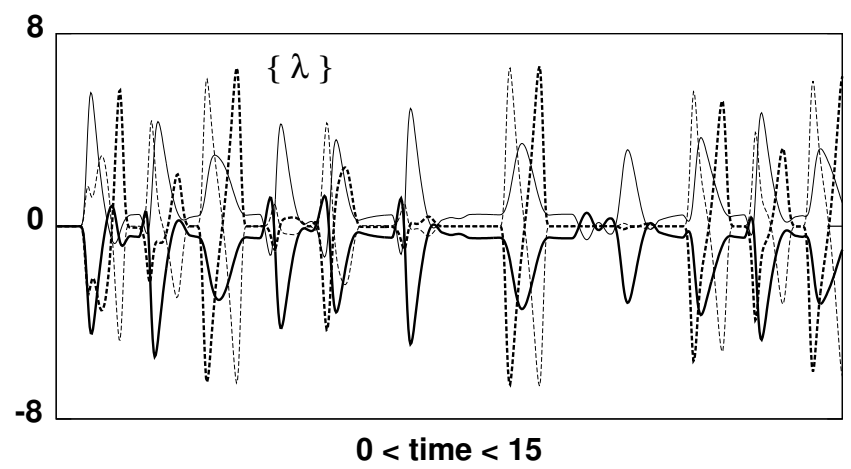

Fig. 3. The four local Lyapunov Exponents for the cell model illustrating exponent "pairing"

There is a set of first-order ordinary differential equations equivalent to the Gram-Schmidt procedure just described in the small timestep limit [5], $d t \longrightarrow 0$ :

$$
\begin{gathered}
\dot{\delta}_{1}=D \cdot \delta_{1}-\lambda_{11} \delta_{1} ; \\
\dot{\delta}_{2}=D \cdot \delta_{2}-\lambda_{21} \delta_{1}-\lambda_{22} \delta_{2} ; \\
\dot{\delta}_{3}=D \cdot \delta_{3}-\lambda_{31} \delta_{1}-\lambda_{32} \delta_{2}-\lambda_{33} \delta_{3} ; \\
\dot{\delta}_{4}=D \cdot \delta_{4}-\lambda_{41} \delta_{1}-\lambda_{42} \delta_{2}-\lambda_{43} \delta_{3}-\lambda_{44} \delta_{4} .
\end{gathered}
$$

Here the matrix $D$ describes the effect of the perturbations $\{\delta\}$ on the unconstrained motion of the vectors. The ten Lagrange multipliers $\left\{\lambda_{i \geq j}\right\}$ vary with time so as to maintain the ten orthonormality constraints, $\left\{\delta_{i} \cdot \delta_{j} \equiv \delta^{2} \delta_{i j}\right\}$. The diagonal Lagrange multipliers in these differential equations are identical to the local Lyapunov exponents, $\lambda_{i i} \equiv \lambda_{i}(t)$. It is easy to show that the differential equations are perfectly timereversible (in the sense that the coordinates are unchanged while the momenta and Lagrange multipliers change sign). This apparent but illusory time symmetry is broken, even for simple systems such as our one-particle cell model. It is also easy to show that exactly the same ten Lagrange multipliers result if the basis vectors are used to describe the virtual growth rates of a two-trajectory length, a three-trajectory equilateral triangle, and a four-trajectory regular tetrahedon.

For relatively short times solutions of this simple dynamical system can be generated with Runge-Kutta integration. The longtime irreversibility of such Runge-Kutta integrations is due to the cumulative growth of single-timestep errors. These local errors are proportional to $d t^{5}$ times the fifth time derivative of the phase-space variables. To avoid the resulting longtime irreversibility the dynamics can instead be generated as an ordered series of coordinate values $\left\{\left(x_{t}, y_{t}\right)\right\}$ using a somewhat less accurate but completely "bit-reversible" in- teger algorithm for the reference trajectory. Among them, Levesque and Verlet's third-order algorithm [10, 11] is certainly the simplest:

$$
\left\{q_{t+d t}-2 q_{t}+q_{t-d t} \equiv\left[F_{t} d t^{2} / m\right]_{\text {Integer }}\right\} .
$$

Rather than the phase variables $\left\{q_{t}, p_{t}\right\}$ two sets of adjacent coordinate values $\left\{q_{t}, q_{t \pm d t}\right\}$ are required to start the Levesque-Verlet algorithm. Here the coordinates and their second differences are all evaluated as (large) integers. The resulting bit-reversible reference trajectory can be extended infinitely far into the future or the past without any need to store the trajectory. A set of momenta corresponding to the coordinates,

$$
\left\{\ldots, p_{t-d t}, p_{t}, p_{t+d t}, \ldots,\right\},
$$

and, like the coordinates, with third-order accuracy in $d t$, can be defined as follows [9]:

$$
\begin{aligned}
p_{t} & \equiv(4 / 3)\left[q_{t+d t}-q_{t-d t}\right] /(2 d t) \\
& -(1 / 3)\left[q_{t+2 d t}-q_{t-2 d t}\right] /(4 d t) .
\end{aligned}
$$

The nearby satellite trajectories are generated with the usual Runge-Kutta integration. By using 16-byte integers the accuracy of the integer-algorithm's reference trajectory can be made to match that of a double-precision floating-point simulation.

A practical approach uses bit-reversible integration for the reference trajectory and fourth-order Runge-Kutta integration for the four nearby satellite trajectories. At the end of each timestep we use Gram-Schmidt orthonormalization, keeping the lengths of the four "offset vectors" fixed $\left\{\left|r_{s}-r_{r}\right|=\delta\right\}$ and their directions orthogonal. The accuracy of the Lyapunov spectrum depends (relatively weakly) upon the timestep $d t$ and the vector length $\delta$. A convenient initial condition,

$$
\left\{x, y, p_{x}, p_{y}\right\}=\{0.0,0.0,0.6,0.8\},
$$

with total energy $E=K+\Phi=(1 / 2) \geq \sum \phi$, guarantees that the moving particle can get no closer to any of its four fixed neighbors than a distance $r_{\min }=\sqrt{\left(1-(1 / 2)^{1 / 4}\right)}=0.3988779$. At the end of each timestep the periodic boundary conditions are applied to ensure that the moving disk stays within its periodic cell. A million timestep simulation using the classic fourth-order RungeKutta integrator for the reference trajectory with $d t=0.0002$ exhibits an energy loss less than one part in $10^{13}$.

Long time energy loss can be avoided entirely, and the numerical trajectory can be made precisely time-reversible, by using Levesque and Verlet's bit-reversible integrator. That algorithm requires a pair of subroutines mapping the floatingpoint interval $\{-2<$ float $<+2\}$ onto the integer interval $\{-M<$ int $<+M\}$ :

int $=$ float $* \mathrm{M} / 2.0 \mathrm{~d} 00 \longleftrightarrow$ float $=2.0 \mathrm{~d} 00 *$ int $/ \mathrm{M}$. 
We choose $M=10^{16}$ so that the precision of the bitreversible simulation is comparable to that of a typical doubleprecision fourth-order Runge-Kutta simulation.

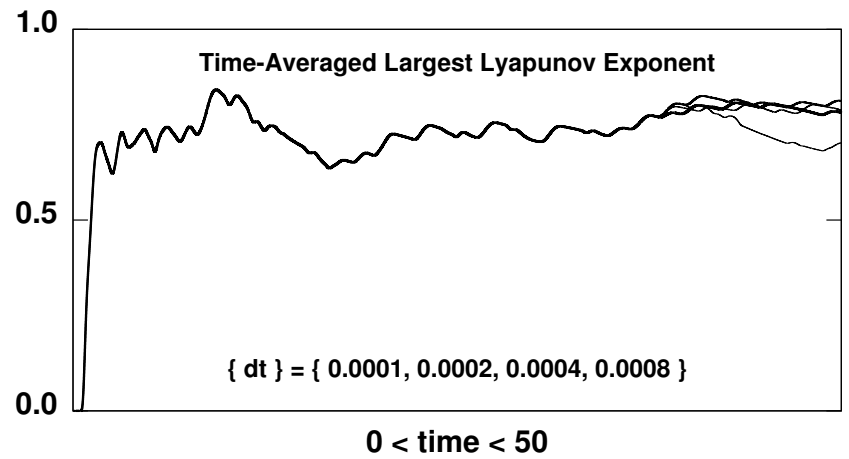

Fig. 4. Dependence of the local Lyapunov exponent on the timestep $d t$

Two initially different offset vectors come to agree - to six-figure accuracy - after propagating for a time of order 20 . Figure 4 shows the early stages of convergence of the nonzero Lyapunov exponent for four different timesteps, 0.0001, $0.0002,0.0004$, and 0.0008 . From the visual standpoint the results are identical until a time of order 40, where Lyapunov instability causes the four trajectories to separate. This longtime-averaged Lyapunov spectrum, $\{+0.7,0,0,-0.7\}$ is, as we would expect, perfectly consistent with the two-system offset vector calculation documented in Figure 2.

The more complicated simulation of Figure 3, giving the whole spectrum, involves solving 20 ordinary differential equations - four of them describing the reference trajectory and 16 more describing its four satellite trajectories. This onebody cell-model problem is an excellent warmup exercise for the many-body problems described in what follows in the next two Sections. These upcoming many-body examples are more complex, in that exponent pairing is a transient (and therefore only approximate) phenomenon. The loss of pairing is evidently associated with dynamical events that appear irreversible, brought about by the choice of inhomogenous out-of-the-ordinary initial conditions.

\section{INELASTIC COLLISIONS OF TWO COLD HEXAGONAL CRYSTALLITES}

Thermodynamic irreversibility occurs whenever mechanical energy is dissipated into heat. We wish to see how such thermodynamic irreversibility is reflected in the Lyapunov instability of atomistic simulations of conservative Hamiltonian mechanics. To begin we will consider a simple demonstration of irreversible behavior, the inelastic collision of two cold seven-atom crystallites to form a single hotter 14-body drop. Our first experience with this general problem type, in 1990, was intended to measure the "coefficient of restitution" for two bouncing balls. But the balls refused to bounce, instead fusing, so as to form a single ball, just as in the present work. The earlier two-ball work is mentioned, and illustrated, in Reference 7. A recent four-ball analog appears on page 96 of (the second [2012] edition) of Reference 1. This same combination of the many-body embedded-atom potential with the repulsive core potential is useful for modeling surfaces and other lattice defects, as well as the dynamics of plastic flows [13]. For the problems considered here the vapor pressure of the coalesced balls is so low that no special spatial boundary conditions are required to contain all the particles.

Each particle has unit mass. In addition to the repulsive pair forces derived from the $\left(1-r^{2}\right)^{4}$ pair potential, we add on a longer-range attractive smooth-particle potential based on the deviations of the individual particle densities from unity, as calculated from Lucy's smooth-particle weight function [1], with a range $h=3.5$ :

$$
\begin{gathered}
\Phi(\{\rho\}) \equiv \sum_{i=1}^{14}(1 / 2)\left(\rho_{i}-1\right)^{2} ; \rho_{i}=\sum_{j=1}^{14} w\left(\left|r_{i}-r_{j}\right|\right) ; \\
w_{\text {Lucy }}(r<h=3.5)=\left(5 / \pi h^{2}\right)[1+3 z][1-z]^{3} ; \\
z \equiv(r / h) .
\end{gathered}
$$

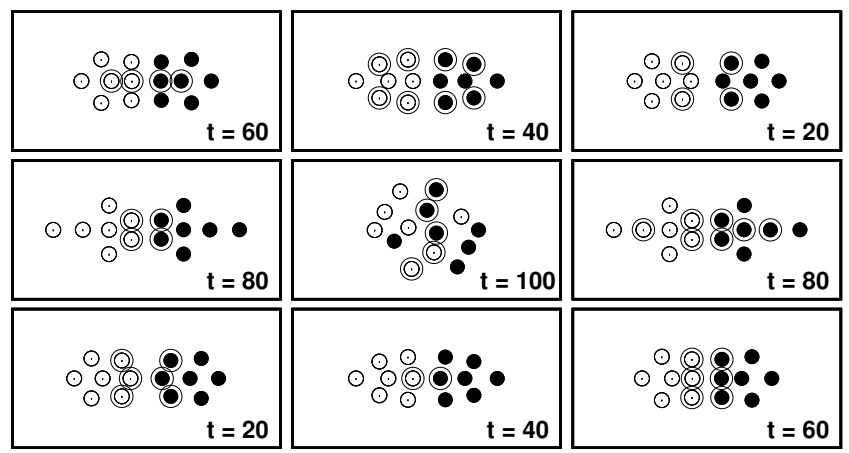

Important Particles Forward and Backward

Fig. 5. Important particles in a 14-particle inelastic collision timereversed at $t=100$

Lucy's weight function is normalized to reflect the local density, with

$$
\int_{0}^{h} 2 \pi r w(r) \equiv 1
$$

The contribution of the smooth-particle potential to the equations of motion is

$$
\begin{aligned}
\ddot{r}_{i} & =\sum_{j}\left[\left(1-\rho_{i}\right) \nabla_{i} w_{i j}+\left(1-\rho_{j}\right) \nabla_{i} w_{i j}\right]= \\
& =\sum_{j}\left(2-\rho_{i}-\rho_{j}\right) \nabla_{i} w_{i j} .
\end{aligned}
$$


Figure 5 shows a series of snapshots of two colliding 7-particle hexagons with the time reversed at $t=100$.

Figures 6 and 7 show similar series of snapshots for two 37-particle hexagons. Just as before, the initial velocities are $p_{x}=+0.1$ for those particles in the left hexagon and $p_{x}=-0.1$ for those at the right. In these figures particles making an above-average contribution to the local Lyapunov exponent $\lambda_{1}(t)$ are distinguished by an extra circular ring for emphasis. Note particularly that in the forward-in-time motions the leading-edge particles contribute most to instability. In the reversed collision, with the drop separating into two hexagons, the cooperative motion of the interior particles is more important to the stability. In the initial least-energy cold configuration for Figure 5 the nearest-neighbor spacing is 0.861121270463 and the seven-body crystal's comoving energy is 0.639029609388 . The energy is positive due to the contribution of the attractive potential, which vanishes at a density of unity, not zero. In Figures 6 and 7 we have chosen a stronger repulsive pair potential, $10\left(1-r^{2}\right)^{4}$ rather than $\left(1-r^{2}\right)^{4}$, in order to compensate somewhat for the layering tendency of the embedded-atom interaction.

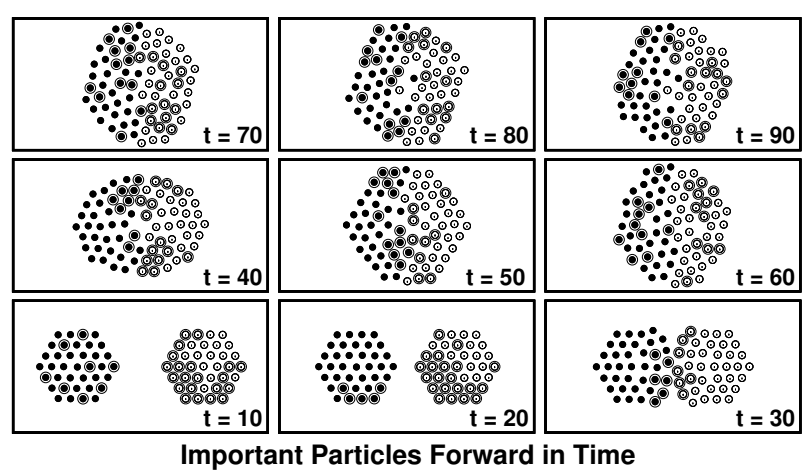

Fig. 6. Important particles are emphasized in the collision of two 37-particle crystallites

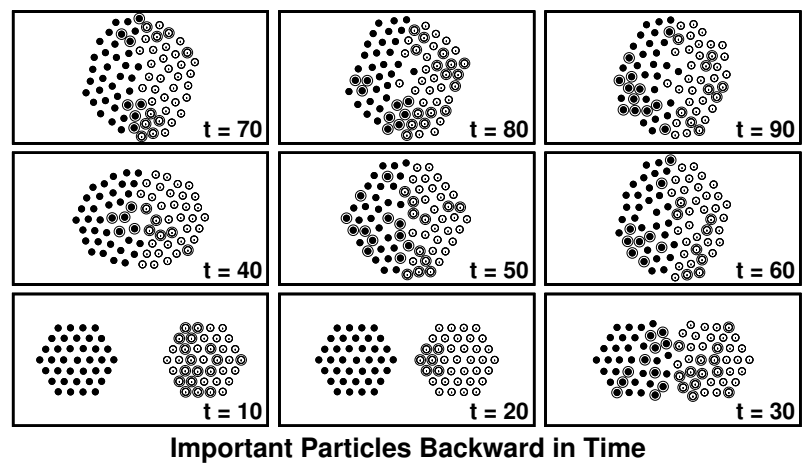

Fig. 7. Same as Figure 6 but with the bit-reversible trajectory processed backward in time
We began by investigating such two-hexagon collisions with the classic fourth-order Runge-Kutta integrator. Although the energy changes can be made negligible for elapsed times of several hundred, Lyapunov instability eventually spoils the details of a "reversed" Runge-Kutta trajectory, and in a much shorter time, of order 25. See again Figure 4. Quadruple precision would simply double this time, to 50. Energy conservation provides no hint of this trajectory irreversibility. Choosing a timestep of $d t=0.001$ conserves the energy to an accuracy of twelve digits over the course of a 600,000 timestep run. But the time reversibility is effectively destroyed much sooner, at about 25,000 timesteps.

To maintain precise time reversibility in our Lyapunov computations, we used the Levesque-Verlet bit-reversible integrator. Figures 5-7 are based on bit-reversible reference trajectories with Runge-Kutta satellite trajectories orthonormalized at each timestep. For the same number of force evaluations per unit time the bit-reversible timestep could be made four times smaller:

$$
d t_{\text {bitrev }}=(1 / 4) d t_{\mathrm{RK} 4}=0.00025 .
$$

But for simplicity we have used $d t=0.001$ for both integrators.

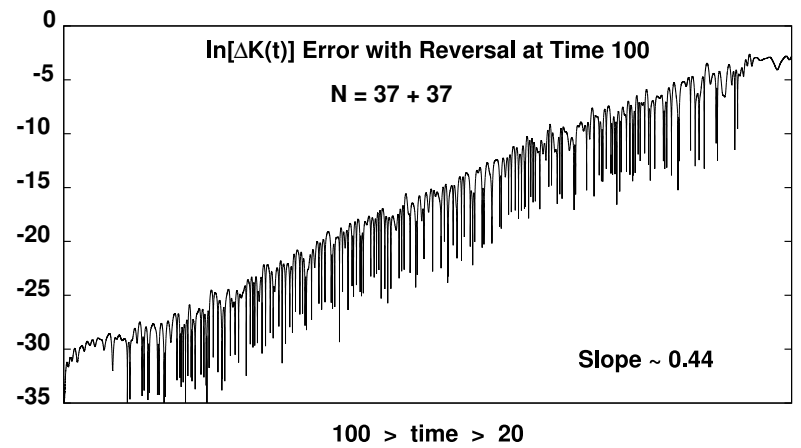

Fig. 8. Exponential growth of kinetic energy error after time-reversal at time 100

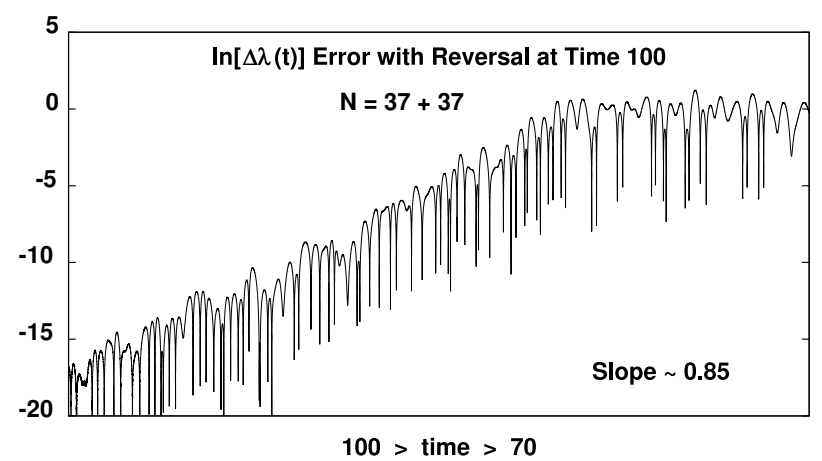

Fig. 9. Exponential growth of Lyapunov exponent error after timereversal at time 100 


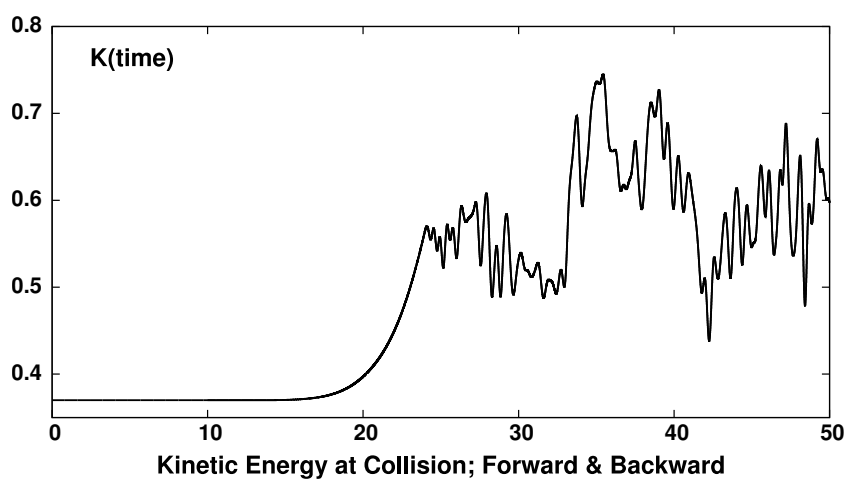

Fig. 10. Variation of Kinetic energy (initially $37 \times 2 \times 0.005$ ) during the bit-reversible inelastic collision of two 37-particle crystallites

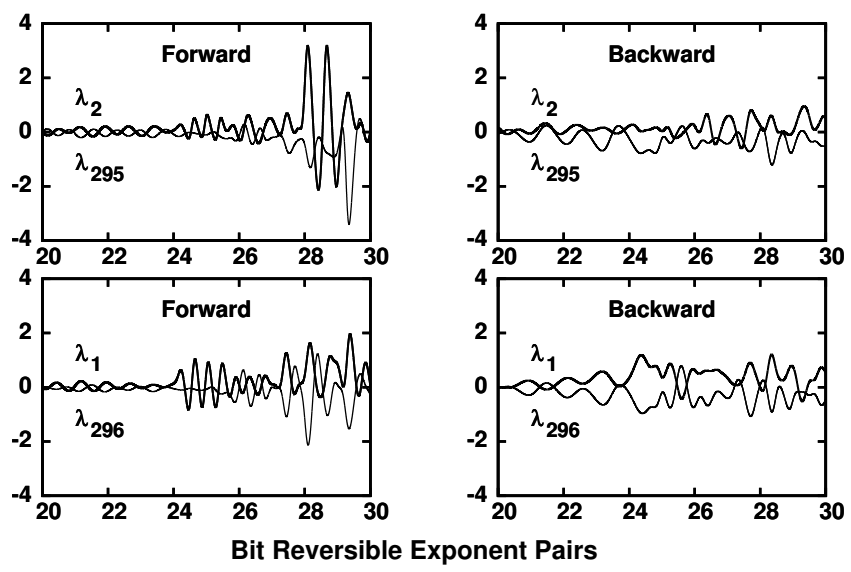

Fig. 11. Lack of pairing relationships between the first and last Lyapunov exponents during the bit-reversible simulation of a 74-body inelastic collision

Figures 8 and 9 compare the Runge-Kutta and bitreversible calculations of energy and the largest Lyapunov exponent for the $37+37$ particle problem. Note again that the local Lyapunov exponent is a much more sensitive test of trajectory accuracy than is the energy. The comparison also shows that either algorithm, Runge-Kutta or bit-reversible, can be used for simulations that are not too long. Figure 10 shows the thermalization of the kinetic energy as the two hexagons merge to form a warm ball. At about time 30 the coalescence is complete. The remaining dynamics consists of relatively featureless thermal motion. In Figure 11 we show a portion of the time-dependence of the 1-296, and 2-295 pairs of local Lyapunov exponents, both forward and backward in time. From the visual standpoint simulations using a bit-reversible reference trajectory are indistinguishable from those using Runge-Kutta integration, with time reversed, $+d t \rightarrow-d t$, at a time of 25 . These results show very clearly that pairing is not a general phenomenon. The more negative exponents react earlier, and more strongly, to the collision process than do the more positive ones.
During the progress of the collision we can locate the "important" particles, those making above average contributions to the length of the instability offset vector $\delta_{1}(t)$. As one might expect, the particles on the leading edges of the crystallites are the first to feel the collision. In the time-reversed motion other particles become important. This is interesting! We will detail this lack of time symmetry in a larger and more complex coalescence problem in the next Section.

\section{INELASTIC COLLISION OF TWO LARGER CRYSTALLITES}

In two dimensions problems with a few hundred particles are already large enough to suggest continuum flows. Figures 12 and 13 show a series of forward and reversed snapshots from the collision of two cold 400-particle crystallites with the same repulsive pair potential and the same attractive embedded-atom potential as in the 74-particle problem of the last Section. The initial state uses two copies of a 400-particle crystallite generated by the relaxation of a $20 \times 20$ square structure. The relaxation providing initial conditions for all these problems is easily carried out by including viscous forces, $\{-(p / \tau)\}$, in the dynamics. For simplicity, coordinates and velocities for a second crystallite were chosen to satisfy inversion symmetry relative to the first:

$$
\begin{gathered}
\left\{x^{\text {left }}(i)+x^{\text {right }}(i)=0=y^{\text {left }}(i)+y^{\text {right }}(i)\right\} ; \\
\left\{p_{x}^{\text {left }}(i)=+0.10 ; p_{x}^{\text {right }}(i)=-0.10\right. \\
\left.p_{y}^{\text {left }}(i)=0.00=p_{y}^{\text {right }}(i)\right\} .
\end{gathered}
$$

Just as in the smaller cases this 800-particle problem exhibits two different local Lyapunov spectra, one going forward in time and the other going backward. The "important particles" are indicated by central dots in the figures. Here the reference trajectory is bit-reversible so that the forward and backward particle coordinates agree to the very last bit. The local exponents and vectors at a time $t$ can be determined accurately by analyzing the trajectory segment from $t-30$ to $t+30$.

Figure 12 shows that forward in time the important particles are located in the collision region, where the two crystallites first deform. Backward in time (Figure 13) a complex collective synchronized motion of the crystallites is required to regain the zero-temperature structures. This "unlikely" motion is localized in the necking region of the coalesced crytals. This symmetry-breaking provides an "Arrow of Time" for the coalescence problem. The geometric features of the Lyapunov instability, given by the offset vectors, are qualitatively different in the forward and reversed time directions. 

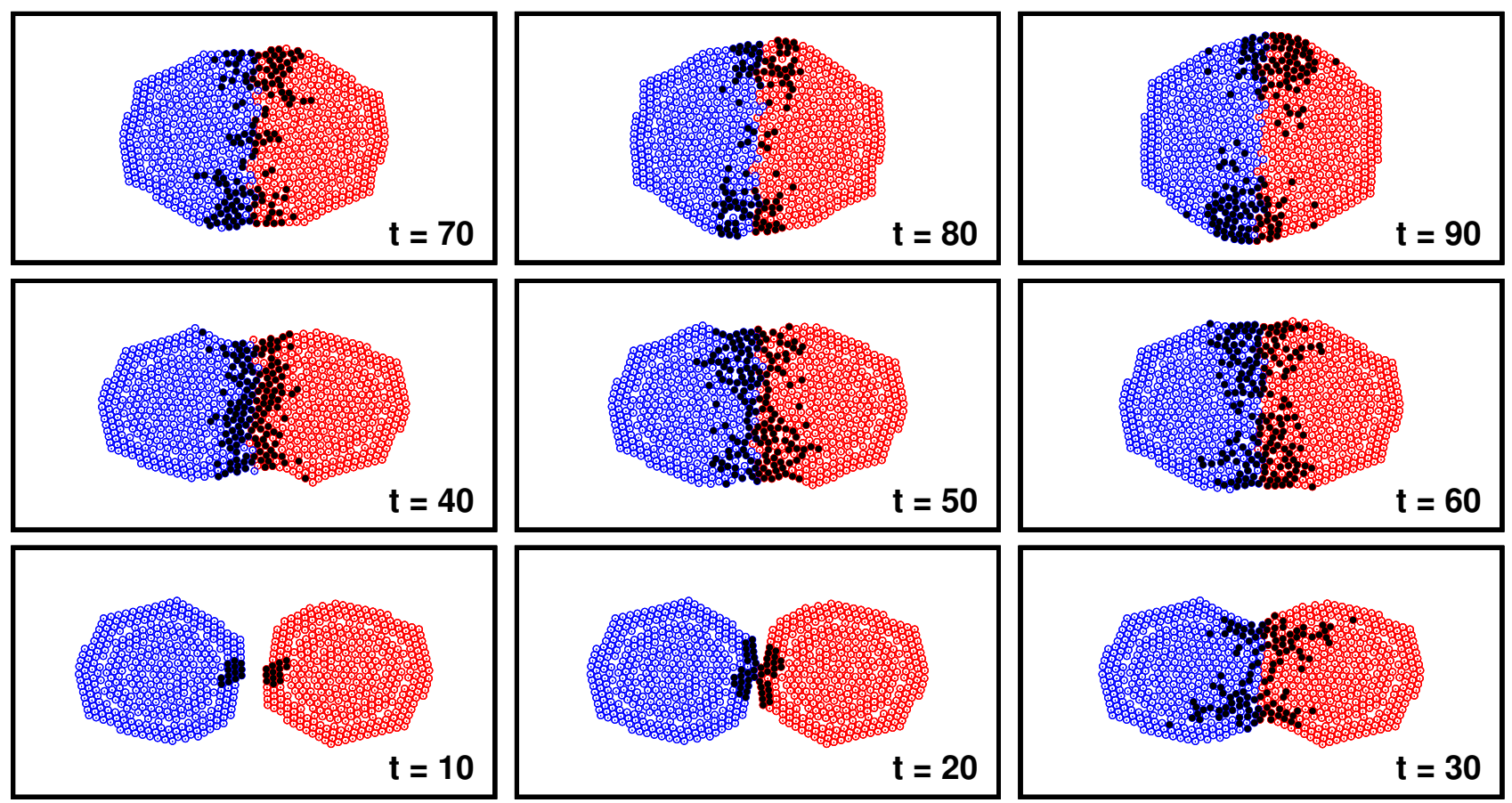

Forward Times 10 to $90 ; \mathrm{dt}=\mathbf{0 . 0 0 1}$

Fig. 12. Important particles (black) during the collision of two 400-particle crystallites
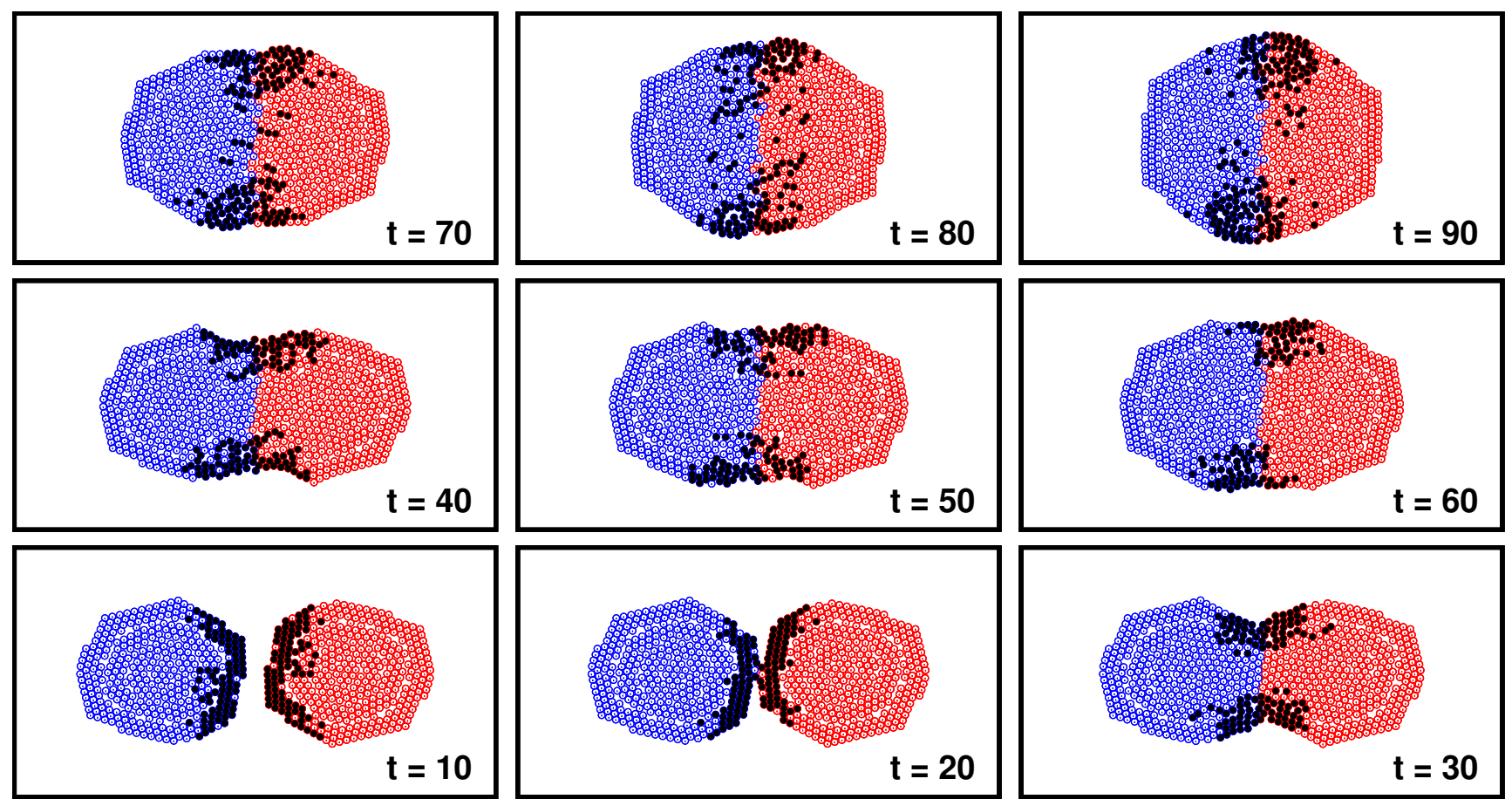

Backward Times 10 to $90 ; \mathrm{dt}=0.001$

Fig. 13. Important particles during the (bit-reversibly reversed) collision of Figure 12. Note the qualitative difference to Figure 12 with precisely identical coordinates at corresponding times 
This same symmetry-breaking is exactly the same for the recently-popularized "covariant vectors" [10], which are a modified approach to describing phase-space instability. The first and last covariant vectors correspond to the forward and reversed versions of our $\delta_{1}(t)$ vectors. Prediction of symmetry-breaking, both of the positive and negative members of each exponent pair, as well as the symmetry-breaking distinguishing the offset vectors forward and backward in time, requires a nonlinear analysis, as all of the equations for the reference and satellite trajectories are strictly timereversible.

\section{CONCLUSION AND SUMMARY}

The observed irreversibility of simple nonequilibrium processes includes many examples from gas theory as well as both transient and steady flows of condensed matter. Our coalescence problems are good examples of irreversible processes. Deterministic time-reversible microscopic models are available to simulate many such problems. How does timereversible microscopic mechanics give rise to this variety of irreversible nonlinear macroscopic behavior?

Boltzmann's H Theorem answers this question for dilute gases [14]. He showed that the Maxwell-Boltzmann velocity distribution is the overwhelmingly probable result of "uncorrelated" collisions, collisions with randomly-chosen impact parameters. The thermostatted forms of reversible mechanics developed in the 1970s and 1980s provided a different explanation [8], useful for understanding condensed matter simulations of nonequilibrium steady states. With the new forms of mechanics the irreversibility of nonequilibrium flows could be traced to their extreme (fractal) rarity and to their stability, relative to their time-reversed twins, in phase space. Thus the entropy of nonequilibrium macroscopic states, as measured by the (logarithm of) the number of corresponding microscopic phase-space states, is both singular and divergent [8]. This fractal character is well-established for many simple model systems [1,2]. In modelling a typical stationary timereversible flow (like thermostatted plane Couette shear flow or steady Fourier heat conduction) a fractal attractor forms in phase space, with a negative Lyapunov sum giving the exponential rate of phase-volume collapse. The time-reversed repellor, with its unstable (positive) Lyapunov sum, provides the source for phase-space probability flow to the fractal sink, a strange attractor. The fractal nature of such flows corresponds to the extreme rarity of nonequilibrium steady states. All such thermostatted simulations require a nonHamiltonian dynamics in order to generate and account for the concentration of phase-space probability on a fractal.

The present examples are quite different. There are neither statistical collisions nor fractal distributions, though there is certainly a coarse-grained macroscopic entropy increase, invisible according to Liouville's Theorem, from minus infinity in the cold crystallites, to a positive equilibrium value in the resulting equilibrated coalesced state. Where does Time's Arrow come in? The futures and the histories of the forward (or primary) and reversed flows are (almost) exactly the same from the standpoint of configurations $\{q\}$. The "almost" reminds us of the difficulty in constructing a primary trajectory in the direction that violates the Second Law! Regardless, two kinds of pairing, [i] with any positive Lyapunov exponent paired to a corresponding negative one, and [ii] with any forward Lyapunov exponent paired to a corresponding backward one, are both consistent with the time-reversible Hamiltonian equations of motion. But the stabilities of the time-reversed motion equations are complicated, in their model dependence and in their time dependence. For flows which are relatively simple, like the cell model, the motions in the two time directions can fail to distinguish the Lyapunov instability's dependence on the past from its symmetry with the future. More complex flows, like the colliding crystallites, or shockwaves, come instead to reflect the past rather than the future. In these cases knowledge of $\delta_{1}$ automatically gives the direction in which the flow is developing.

We have seen that the Lyapunov instabilities inherent in the dynamics always reflect the past rather than the future. The delay between cause and effect is the same as that observed in atomistic shockwave simulations where the stress lags the strainrate and the heat flux lags the temperature gradient $[9,15]$. The forward-backward symmetry of the microscopic motion equations does not carry through to the macroscopic diagnostics of the motion.

Although the dynamics is symmetric in the time the stability of that dynamics is not. The morphology of the exponents provides a clue as to whether or not we are looking at an equilibrium system. Whenever the past is quite different to the future this lack of symmetry can be seen in the local Lyapunov spectrum. The lack of pairing and the inhomogeneity of the local Lyapunov exponents needs to be related to macroscopic entropy production. Liouville's Theorem shows that the Lyapunov spectrum, which sums to zero with Hamiltonian mechanics, is inconsistent with macroscopic entropy change. On the other hand systems like our colliding crystallites, manifesting a failure of the past and future to pair, may come to suggest new metrics for the separation from equilibrium and its evolution.

\section{Acknowledgments}

We are particularly grateful to Kris Wojciechowski for stimulating this work and to Franz Waldner, Marc Meléndez Schofield, Vitaly Kuzkin, and Harald Posch for their patient comments and suggestions.

\section{References}

[1] Wm.G. Hoover and C.G. Hoover, Time Reversibility, Computer Simulation, Algorithms, and Chaos (World Scientific, Singapore, 2012). 
[2] Wm.G. Hoover, Computational Statistical Mechanics (Elsevier Science, 1991), available free of charge at our website [ www.williamhoover.info ].

[3] S.D. Stoddard and J. Ford, Numerical Experiments on the Stochastic Behavior of a Lennard-Jones Gas System, Physical Review A 8, 1504-1512 (1973).

[4] G. Benettin, L. Galgani, A. Giorgilli and J.M. Strelcyn, Lyapunov Characteristic Exponents for Smooth Dynamical Systems and for Hamiltonian Systems; a Method for Computing All of Them, Meccanica 15, 9-30 (1980).

[5] W.G. Hoover and H.A. Posch, Direct Measurement of Lyapunov Exponents, Physics Letters A 113, 82-84 (1985),

[6] H.A. Posch, Wm.G. Hoover and F.J. Vesely, Canonical Dynamics of the Nosé Oscillator: Stability, Order, and Chaos, Physical Review A 33, 4253-4265 (1986).

[7] Wm.G. Hoover, C.G. Hoover, I.F. Stowers, A.J. De Groot and B. Moran, Simulation of Mechanical Deformation via Nonequilibrium Molecular Dynamics, in Microscopic Simulations of Complex Flows, Edited by Michel Mareschal (Volume 236 of NATO Science Series B, Plenum Press, 1990).

[8] B.L. Holian, Wm.G. Hoover and H.A. Posch, Resolution of Loschmidt's Paradox: the Origin of Irreversible Behavior in Reversible Atomistic Dynamics, Physical Review Letters 59, 10-13 (1987).

[9] Wm.G. Hoover and C.G. Hoover, Time's Arrow for Shockwaves; Bit-Reversible Lyapunov and Covariant Vectors ; Symmetry Breaking, Computational Methods in Science and Technology 19(2), 69-75 (2013).
[10] M. Romero-Bastida, D. Pazó, J.M. Lopéz and M.A. Rodríguez, Structure of Characteristic Lyapunov Vectors in Anharmonic Hamiltonian Lattices, Physical Review E 82, 036205 (2010).

[11] D. Levesque and L. Verlet, Molecular Dynamics and Time Reversibility, Journal of Statistical Physics 72, 519-537 (1993).

[12] J.O. Hirschfelder, C.F. Curtiss and R.B. Bird, Molecular Theory of Gases and Liquids, John Wiley \& Sons, Incorporated (New York, 1954).

[13] S.M. Foiles, M.I. Baskes and M.S. Daw, Embedded-AtomMethod Functions for the FCC Metals $\mathrm{Cu}, \mathrm{Ag}, \mathrm{Au}, \mathrm{Ni}, \mathrm{Pd}, \mathrm{Pt}$, and their Alloys, Physical Review B 33, 7983-7991 (1986).

[14] J.L. Lebowitz, Boltzmann's Entropy and Time's Arrow, Physics Today 46, 32-38 (September, 1993).

[15] F.J. Uribe, Wm.G. Hoover and C.G. Hoover, Maxwell and Cattaneo's Time-Delay Ideas Applied to Shockwaves and the Rayleigh-Bénard Problem, Computational Methods in Science and Technology 19(1), 5-12 (online January 2013).

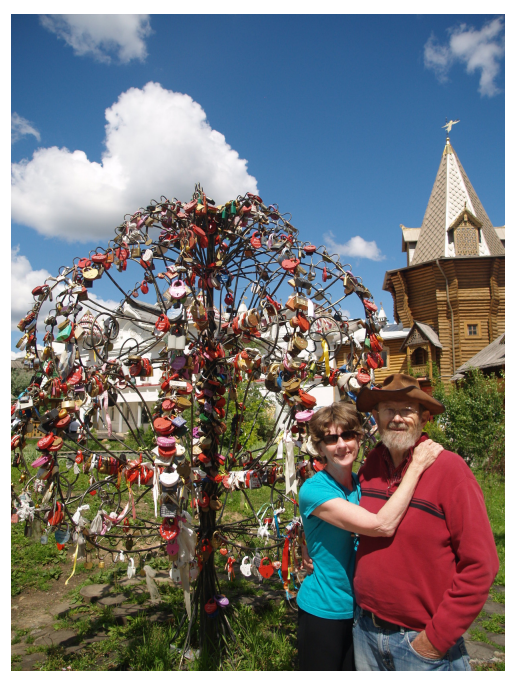

William G. Hoover, Carol G. Hoover, Carol [PhD in Applied Science, University of California, 1978] met Bill at the University of California's Davis/Livermore campus where Bill [PhD University of Michigan, 1961] taught a variety of graduate courses in addition to the core courses in Statistical Mechanics and Kinetic Theory. More than a decade later they met socially, married, and worked together on a one-year research leave/sabbatical at Yokohama with Shuichi Nosé, Toshio Kawai, Taisuke Boku, and Sigeo Ihara. The Hoovers worked at the Lawrence Livermore National Laboratory and the University of California until 2005, when they built a new home in the cattle-ranching community of Ruby Valley, in northeastern Nevada. Their research continues there, with much of it summarized in their recent book, Time Reversibility, Computer Simulation, Algorithms, and Chaos [World Scientific Publishers, Singapore, 2012]. Bill's other books, on Molecular Dynamics, Computational Statistical Mechanics, and SPAM [Smooth Particle Applied Mechanics] are all available free at [http://williamhoover.info]. 